\title{
Rab3 Superprimes Synaptic Vesicles for Release: Implications for Short-Term Synaptic Plasticity
}

\author{
Oliver M. Schlüter, ${ }^{2,3}$ Jayeeta Basu, ${ }^{1,2}$ Thomas C. Südhof, ${ }^{4}$ and Christian Rosenmund ${ }^{1,2}$ \\ ${ }^{1}$ Departments of Neuroscience and Molecular and Human Genetics, Baylor College of Medicine, Houston, Texas 77030, Max-Planck-Institute for \\ ${ }^{2}$ Biophysical Chemistry and ${ }^{3}$ Experimental Medicine, 37075 Göttingen, Germany, and ${ }^{4}$ Center for Basic Neuroscience, Department of Molecular Genetics, \\ and Howard Hughes Medical Institute, The University of Texas Southwestern Medical Center, Dallas, Texas 75390-9111
}

Presynaptic vesicle trafficking and priming are important steps in regulating synaptic transmission and plasticity. The four closely related small GTP-binding proteins Rab3A, Rab3B, Rab3C, and Rab3D are believed to be important for these steps. In mice, the complete absence of all Rab3s leads to perinatal lethality accompanied by a $30 \%$ reduction of probability of $\mathrm{Ca}^{2+}$-triggered synaptic release. This study examines the role of Rab3 during $\mathrm{Ca}^{2+}$-triggered release in more detail and identifies its impact on short-term plasticity. Using patch-clamp electrophysiology of autaptic neuronal cultures from Rab3-deficient mouse hippocampus, we show that excitatory Rab3deficient neurons display unique time- and frequency-dependent short-term plasticity characteristics in response to spike trains. Analysis of vesicle release and repriming kinetics as well as $\mathrm{Ca}^{2+}$ sensitivity of release indicate that Rab3 acts on a subset of primed, fusion competent vesicles. They lower the amount of $\mathrm{Ca}^{2+}$ required for action potential-triggered release, which leads to a boosting of release probability, but their action also introduces a significant delay in the supply of these modified vesicles. As a result, Rab3-induced modifications to primed vesicles causes a transient increase in the transduction efficacy of synaptic action potential trains and optimizes the encoding of synaptic information at an intermediate spike frequency range.

Key words: synaptic transmission; hippocampus; exocytosis; vesicle trafficking; release probability; GTP binding proteins

\section{Introduction}

Rab proteins are the largest family of ras-related GTPases (Pereira-Leal et al., 2001). These are known to act as directional molecular switches at all membrane trafficking events in eukaryotic cells. They are localized on the donor membrane and mediate contact with the target acceptor membrane by recruiting their effectors. Identified functions include the recruitment of SNARE complexes (Rab1), tethering factors (Rab5), or motor proteins (Rab6). The most abundant Rab in brain, localized on synaptic vesicles, is Rab3a. Of all mammalian Rab proteins, it shows the highest degree of simililarity to SEC4, which in yeast is essential for exocytosis. Four Rab3 paralogs (Rab3A, Rab3B, Rab3C, and Rab3D) are expressed in the brain (Touchot et al., 1987; Zahraoui et al., 1989; Baldini et al., 1995; Schluter et al., 2002) and are apparently functionally redundant. Although removal of individual Rab3 isoforms does not affect viability or fertility, genetic deletion of all four rab3 isoforms in mice causes perinatal lethality possibly by respiratory failure (Schluter et al., 2004). The function of Rab3 at the synapse is to regulate vesicular release proba-

Received Aug. 22, 2005; revised Dec. 3, 2005; accepted Dec. 5, 2005.

This work was supported by National Institutes of Health Grant NS051262. We thank the Max-Planck Society and Drs. Reinhard Jahn, Nils Brose, and Erwin Neher for their support. C.R. was also a recipient of a Heisenberg fellowship of the German Research Council. We have no competing financial interests.

Correspondence should be addressed to either of the following: Christian Rosenmund, Departments of Neuroscience and Molecular and Human Genetics, Baylor College of Medicine, One Baylor Plaza, Room 833E, Houston, TX 77030, E-mail: rosenmun@bcm.tmc.edu; or Oliver M. Schlüter, Department of Psychiatry and Behavioral Sciences, Stanford University, Stanford, CA 94305, E-mail: oschlue@stanford.edu.

DOI:10.1523/JNEUROSCI.3553-05.2006

Copyright $\odot 2006$ Society for Neuroscience $\quad$ 0270-6474/06/261239-08\$15.00/0 bility. In excitatory hippocampal neurons, removal of all Rab3s lead to a 30 and $40 \%$ reduction of the mean vesicular and synaptic release probability without affecting the number of primed vesicles (Schluter et al., 2004). The documented reduction in the efficiency of synaptic output for single action potentials will likely affect also the dynamics of postsynaptic response amplitudes during spike trains. Although short-term plasticity (STP) characteristics are governed by multiple factors, synaptic release probability $P_{\mathrm{r}}$ has the greatest impact, because synapses with high $P_{\mathrm{r}}$ tend to depress during spike trains, because readily available vesicles are more quickly consumed as they are replenished. Conversely, synapses with low initial release probability tend to facilitate, because the increase of intraterminal $\mathrm{Ca}^{2+}$ helps to release vesicles that were not as easily released during previous action potentials. Other factors that affect STP include feedback activation of presynaptic receptors (von Gersdorff et al., 1997), presynaptic $\mathrm{Ca}^{2+}$ handling (Edmonds et al., 2000; Rozov et al., 2001; Blatow et al., 2003; Sippy et al., 2003), heterogeneous release probability (Trommershauser et al., 2003), $\mathrm{Ca}^{2+}$-dependent $\mathrm{Ca}^{2+}$ channel inactivation (Xu and $\left.\mathrm{Wu}, 2005\right)$, and postsynaptic receptor desensitization (Otis et al., 1996). In addition, spike train-dependent increases of intraterminal $\mathrm{Ca}^{2+}$ can accelerate vesicle priming (Stevens and Wesseling, 1998). To identify the particular phase of Rab3 action on the vesicular release process and to gain insights into the exact mechanism of Rab3-dependent STP via modulation of vesicular release probability, we used quadruple Rab3A, Rab3B, Rab3C, and Rab3D mice and analyzed synaptic transmission in autaptic neuronal cultures derived from 
the hippocampus. We found that Rab3 proteins play a major and unexpected role in short-term synaptic plasticity. Rab3 proteins act to "boost" the apparent $\mathrm{Ca}^{2+}$ sensitivity of a subset of vesicles in the readily releasable pool (RRP), thereby introducing a functional heterogeneity among the primed vesicles. As a result, the reliability of synaptic responses for lowfrequency presynaptic spiking activity is particularly heightened. Among many possible consequences, this may help to increase accuracy of sensory information coding.

\section{Materials and Methods}

Mouse breeding and genotyping. The Rab3A, Rab3B, Rab3C, and Rab3D knock-out (KO) mice were bred and genotyped as described previously (Schluter et al., 2004).

Cell culture. Mouse embryos of embryonic day 19 (E19) Rab3A ${ }^{+/-}$or Rab3A ${ }^{+/-} \mathrm{BCD}^{-1-}$ timed pregnancies were delivered by cesarean section. Hippocampi from the entire litter were isolated and cultured in a chemically defined media (Neurobasal medium A; Invitrogen, San Diego, CA) supplemented with B27 (Invitrogen) on glial microdot islands (Bekkers and Stevens, 1991; Pyott and Rosenmund, 2002). Islands containing single neurons forming recurrent synapses (autapses) were used after $9-16 \mathrm{~d}$ in culture. In all analyses, cultures from the respective $\mathrm{KO}$ mice and their littermate control mice were initially established and later analyzed on the same days to avoid culture artifacts (Fernandez-Chacon et al., 2001).

Electrophysiology. Synaptic transmission was recorded in whole-cell configuration under voltage-clamp at -75 to $-85 \mathrm{mV}$ (Axopatch 200B; Molecular Devices, Union City, CA) at room temperature $\left(\sim 22-24^{\circ} \mathrm{C}\right)$. Synaptic transmission was induced by depolarization to $0 \mathrm{mV}$ for $2 \mathrm{~ms}$, and the triggered synaptic responses were recorded with a sampling rate of $10 \mathrm{kHz}$ filtered at $2 \mathrm{kHz}$. The standard extracellular recording solution contained the following (in mM): $140 \mathrm{NaCl}, 2.4 \mathrm{KCl}, 10 \mathrm{HEPES}, 10$ glucose, $4 \mathrm{CaCl}_{2}, 4 \mathrm{MgCl}_{2}, \mathrm{pH} 7.3$, with an adjusted osmotic strength of 300 mOsm. Patch pipettes $(2.5-3.5 \mathrm{mU})$ were filled with internal solution containing the following (in $\mathrm{mm}$ ): $125 \mathrm{~K}$-gluconate, $10 \mathrm{NaCl}, 4.6 \mathrm{MgCl}_{2}$, 4 ATP-Na $2,0.3$ GTP-Na, 15 creatine phosphate, $20 \mathrm{U} / \mathrm{ml}$ phosphocreatine kinase, 1 EGTA, pH 7.3, also adjusted to $300 \mathrm{mOsm}$. Solutions were applied using a fast flow application system (Pyott and Rosenmund, 2002). Sucrose ( $500 \mathrm{~mm}$ ) was added to the standard extracellular recording solution for the measurement of size and refilling kinetics of the readily releasable vesicle pool. Relative changes in the $\mathrm{Ca}^{2+}$ dependence of EPSC amplitudes were determined by applying an external solution with an initial high release probability $\left(12 \mathrm{~mm} \mathrm{CaCl}, 1 \mathrm{mM} \mathrm{MgCl}_{2}\right)$ and the effect of subsequent reduction of $\mathrm{Ca}^{2+}$ influx on EPSC amplitude was determined by alternating between control solution and solutions containing variable $\mathrm{Cd}^{2+}$ concentrations $(1-100 \mu \mathrm{M})$. In this manner, EPSC amplitude changes caused by possible divalent concentrationdependent alteration in axonal excitability was excluded. All chemicals were purchased from Sigma with the exception of EGTA-AM (Calbiochem, La Jolla, CA).

Data analysis. The synchronous and asynchronous component of release during high-frequency stimulation was determined as described, without normalizing to the first EPSC (Hagler and Goda, 2001; Pyott and Rosenmund, 2002). Statistical analyses were performed using Student's $t$ test, and significance was considered when $p<0.05$. For data fitted with exponential curves, the goodness of fit for each curve fitting was determined by comparing both the $\chi^{2}$ and sum of squared errors.
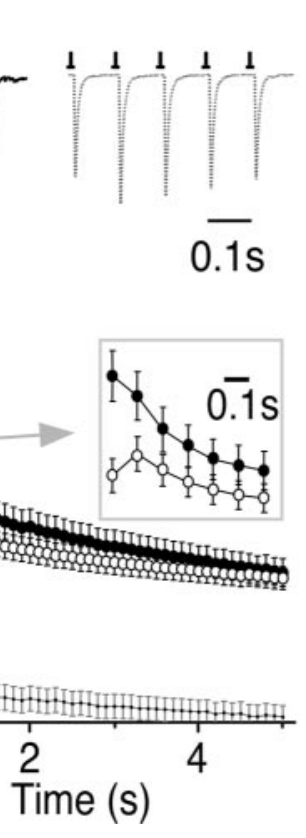

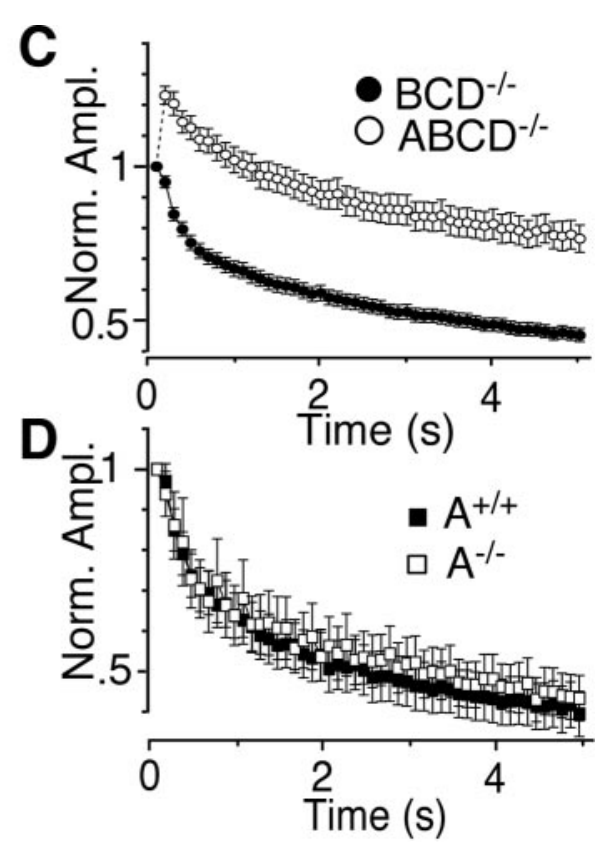

Figure 1. Synaptic responses to repetitive stimulation. $\boldsymbol{A}$, Sample traces for Rab3a-containing (left) and Rab3-deficient exci$\mathrm{Hz}$ spike train compared with the typical depression seen in the wild-type-like Rab3a-containing synapses. $\boldsymbol{B}$, Absolute EPSC mplitude (Ampl.) synaptic responses at $10 \mathrm{~Hz}$ stimulation frequency recorded in $4 \mathrm{~mm} \mathrm{Ca}{ }^{2+}$ in neurons from $\mathrm{Rab}^{2} \mathrm{ABCD}{ }^{-1-}$ responses at higher time resolution. $\boldsymbol{C}$, Normalized EPSC amplitudes from the data set of $\boldsymbol{B}$. $\boldsymbol{D}$, Normalized synaptic responses to 10 KO $(n=20)$ and wild-type control $(n=30)$ mice.

\section{Results}

To study the role of the Rab3-dependent component of release in short-term synaptic plasticity, we used cultured autaptic excitatory hippocampal neurons from Rab3-deficient mice. The triple Rab3BCD KO (BCD ${ }^{-1-}$ ) neurons (hence forward named Rab3a containing) were used as controls for the quadruple $\mathrm{KO}$ Rab3ABCD KO $\left(\mathrm{ABCD}^{-1-}\right)$ neurons (Rab3 deficient) in these experiments, because previous studies have shown that Rab3a (which is the only Rab3 isoform expressed in the triple $\mathrm{KO}$ neurons) is fully capable of maintaining a wild-type like synaptic behavior, and showed no signs of morbidity or impaired survival (Schluter et al., 2004).

\section{Synaptic responses facilitate during action potential trains in Rab3-deficient neurons}

We applied trains of action potentials at a frequency of $10 \mathrm{~Hz}$ and compared the absolute and normalized EPSCs elicited by these action potentials. We found that the EPSC exhibited strong initial facilitation in the quadruple $\mathrm{KO}$, whereas the triple $\mathrm{KO}$ neurons showed normal moderate depression (Fig. $1 A, C$ ). This change in short-term plasticity was not caused by the removal of Rab3a alone, because we observed no facilitation in Rab3A KO neurons compared with their wild-type littermates (Fig. $1 D$ ). To estimate the overall effect of Rab3 deficiency on synaptic output, we plotted the mean absolute EPSC amplitudes of Rab3-deficient and Rab3a-containing neurons (Fig. $1 B$ ). Although the initial synaptic response in Rab3a-containing synapses was $\sim 35 \%$ larger, the responses of the two groups converged to similar amplitudes after approximately five synaptic responses. To reveal the Rab3dependent synaptic component, we subtracted the absolute mean amplitudes at each time point of the Rab3-deficient neurons from the amplitudes of individual Rab3a-containing ones 

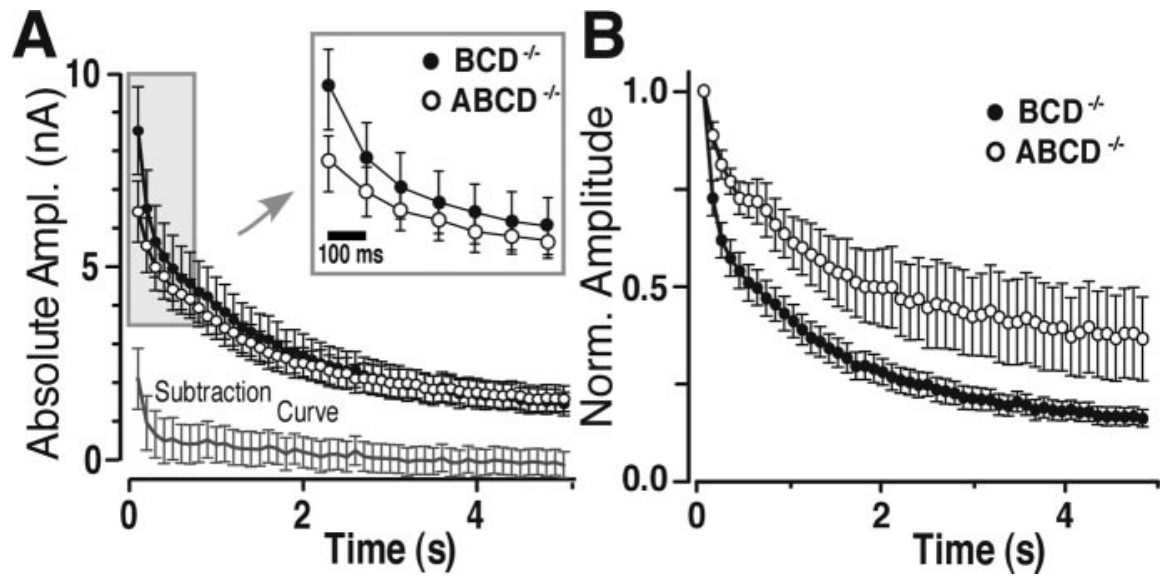

Figure 2. Synaptic responses to repetitive stimulation at increased release probability. $\boldsymbol{A}$, Absolute mean EPSC amplitudes (Ampl.) during $10 \mathrm{~Hz}$ stimulation recorded in $12 \mathrm{~mm}$ extracellular $\mathrm{Ca}^{2+}$ to increase the release probability. The gray curve at the bottom represents the difference in absolute amplitudes between the two genotypes to reveal the Rab3-dependent component. The inset shows the first seven responses at higher time resolution $\left(\mathrm{BCD}^{-1-}, n=20 ; \mathrm{ABC} \mathrm{D}^{-1-}, n=21\right)$. $\boldsymbol{B}$, Same data as in $\boldsymbol{A}$, except that synaptic responses are plotted in normalized (Norm.) terms.

(Fig. $1 B$ ). These data show that Rab3 has a pronounced impact on boosting the initial EPSC amplitude; however, this effect fades quickly after the first five spikes in a train of presynaptic action potentials.

Multiple mechanisms can contribute to changes in STP, including changes in the presynaptic $\mathrm{Ca}^{2+}$ handling (Edmonds et al., 2000; Rozov et al., 2001; Blatow et al., 2003; Sippy et al., 2003). Although we consider this an unlikely scenario in the case of Rab3 based on its structure and its known binding partners, we wanted to verify that the effect of Rab3 removal is independent of intraterminal $\mathrm{Ca}^{2+}$ buffering. We compared $10 \mathrm{~Hz}$ EPSC trains before and after incubation of the neurons with the membranepermeable $\mathrm{Ca}^{2+}$ buffer EGTA (100 $\mu \mathrm{M}$ EGTA-AM; $5 \mathrm{~min}$ ). As expected, the increase in exogenous $\mathrm{Ca}^{2+}$ buffer in the nerve terminal caused a reduction of the initial EPSC amplitude in both groups ( $\sim 20-30 \% ; n=7)$, we also observed enhanced depression in both Rab3a-containing $(n=4)$ and Rab3a-deficient synapses $(n=3)$ (Hagler and Goda, 2001; Rozov et al., 2001), supporting the fact that enhanced $\mathrm{Ca}^{2+}$ buffering affects short-term plasticity. However, although both groups showed more depression under increased buffering, the relative differences between the two groups were maintained (data not shown). This supports the idea that the phenotype associated with the lack of Rab3 is not an exponent of changes in handling of residual $\mathrm{Ca}^{2+}$ but rather a direct effect of Rab3 on the release machinery itself.

\section{Does Rab3 boost release probability in a subset of primed vesicles?}

How is the effect of Rab3 limited to only the first few action potentials during a spike train? From previous experiments, we know that Rab3 removal does not affect the availability of primed vesicles. Among two possibilities, Rab3 may boost release probability by acting on the entire pool of readily releasable vesicles on a temporary time scale or, alternatively, by modifying only a subset of vesicles within the pool. If the entire pool is equally affected, we would not expect that the amplitudes of the two groups would merge within a few action potentials (Fig. $1 B$ ), unless significant depletion had occurred. However, the mean vesicular release probability is only $4.5 \%$ in the Rab3-deficient and 6\% in Rab3acontaining control neurons (Schluter et al., 2004). Therefore, the convergence of the amplitudes within five action potentials is unlikely because of pool depletion. If, however, Rab3 acts on a subset of vesicles and boosts their release probability, then they would preferentially fuse and be depleted during the first few action potentials of the spike train. This would explain the observed transient effect of Rab3. If heterogeneity is an intrinsic property of the vesicles within their subpools, it should persist when the initial release probability is altered.

To test this hypothesis, we increased release probability by elevating extracellular $\mathrm{Ca}^{2+}$ in the external medium (from the regular $4 \mathrm{mM} \mathrm{Ca}^{2+} / 4 \mathrm{mM} \mathrm{Mg}^{2+}$ to $12 \mathrm{~mm}$ $\mathrm{Ca}^{2+} / 1 \mathrm{mM}^{\mathrm{Mg}^{2+}}$ ) and analyzed the relative changes in short-term plasticity characteristics during $10 \mathrm{~Hz}$ trains in the Rab3-deficient synapses versus the Rab3acontaining synapses. Plots of absolute responses confirmed that the Rab3-deficient synapses exhibited a selective loss of synaptic strength during the initial stimuli of the $10 \mathrm{~Hz}$ train, leveling out with the control within a few stimuli (Fig. $2 \mathrm{~A}$ ). As expected (Zucker and Regehr, 2002), synaptic responses depressed much faster and stronger under conditions of increased release probability. However, we still observed that responses in the quadruple $\mathrm{KO}$ neurons show relatively lesser depression than the triple $\mathrm{KO}$ (Fig. 2 B). Thus, the patterns of changes in short-term plasticity in the Rab3-deficient neurons are insensitive to elevations of release probability. Therefore, a global increase of release probability does not lead to a disappearance of the Rab3-deficient phenotype. This observation inclines us to favor the idea of heterogeneity of intrinsic vesicle release properties.

\section{Paired-pulse facilitation reveals additional heterogeneity of Rab3 action}

Because Rab3 may regulate release probability in a subset of vesicles, it would inherently introduce a use-dependent component to synaptic output. To elucidate which time window is most affected by Rab3, we measured short-term plasticity as a function of the frequency of synaptic responses. Most simply, this can be analyzed by varying the interval between two synaptic stimuli and computing the ratio of the second response to the first (Fig. $3 A$ ). We also extended the analysis to a comparison of the 10th and the first response (Fig. 3B).

Interestingly, the loss of Rab3 showed its greatest impact on responses to paired-pulse stimulation when they were applied at intermediate frequencies. Although at interpulse intervals of 20 and $33 \mathrm{~ms}$, factors other than Rab3 dominate the use dependency of the synapse, interpulse intervals of $100 \mathrm{~ms}$ caused an unusually strong increase in the second response in the Rab3-deficient synapses. In contrast, Rab3a containing synapses show at intermediate and long interstimulus intervals more stable responses with only a moderate depression of the synaptic responses, thereby implicating this molecule in stabilizing synaptic output in this frequency range.

Paired-pulse facilitation is inversely proportional to the initial release probability (Zucker and Regehr, 2002). We asked whether the increased facilitation in quadruple KO neurons is solely attributable to the decrease in release probability, affecting all vesicles equally. This would explain the observed phenotype by a simple shift in vesicular release probability. In contrast, if coex- 


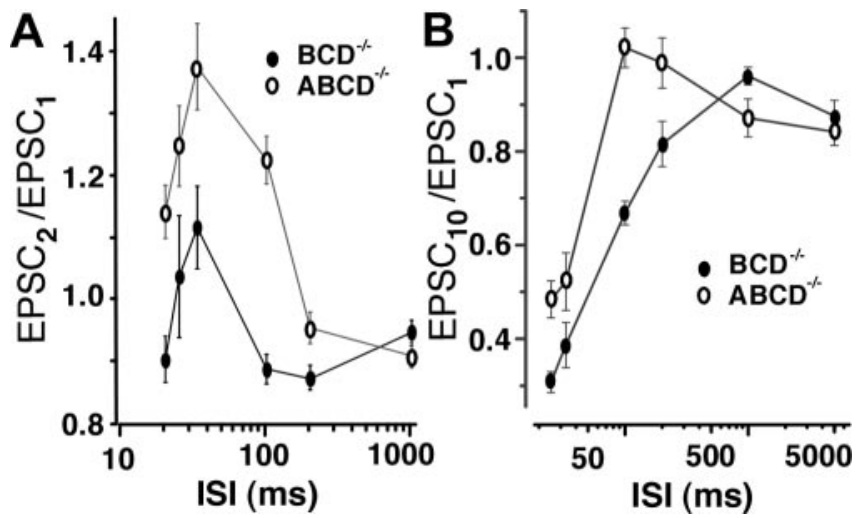

Figure 3. Paired-pulse ratio in Rab3-deficient synapses. $A$, Paired-pulse ratio (ratio of the second to the first synaptic response to two closely spaced action potentials) plotted as a function of the stimulus interval. Experiments were performed in standard extracellular $\mathrm{Ca}^{2+}$ solution (4 $\left.\mathrm{mm} \mathrm{Ca}^{2+} / 4 \mathrm{~mm} \mathrm{Mg}^{2+} ; n=16-182\right) . \boldsymbol{B}$, Ratio of the 10 th synaptic response to the first synaptic response recorded in stimulus trains applied between 0.2 and $50 \mathrm{~Hz}$. Responses are plotted as a function of the interstimulus interval (ISI) based on the stimulation frequency.

isting Rab3a-dependent and Rab3-independent components of release have intrinsically different release probabilities, we would expect that lowering the release probability in all wild-type synapses would not induce the same changes in paired-pulse ratios seen in the Rab3-deficient neurons. To address this issue, we systematically lowered release probability in wild-type neurons to levels equivalent to or lower than that in the Rab3-deficient synapses and compared paired-pulse behavior under these conditions. Release probability was reduced by lowering $\mathrm{Ca}^{2+}$ influx, either by reducing external $\mathrm{Ca}^{2+}$ concentrations from $4 \mathrm{~mm}$ to 2

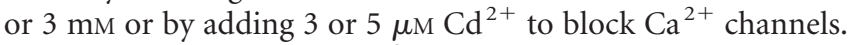
These means of affecting $\mathrm{Ca}^{2+}$ influx have been shown previously to affect presynaptic release in a similar manner (Mintz et al., 1995).

We found that reduction of release probability in wild-type synapses to $54-72 \%$ of the control value barely changed the paired-pulse behavior at $100 \mathrm{~ms}$ interval (Fig. 4). This is in stark contrast to the Rab3-deficient synapses that display at 30\% reduced release probability strong paired-pulse facilitation. At higher frequencies, the difference between Rab3-containing and Rab3-deficient neurons were mostly occluded, most likely because of the prominent effect of classical short-lived facilitation. The discrete effect of Rab3 removal on paired-pulse behavior at intermediate frequencies indicate that the underlying mechanism of Rab3 action cannot be explained by an increase in mean release probability across all vesicles; rather, this effect compliments the idea that a change in the release properties of a subpopulation of vesicles is the underlying cause. As a result, addition of Rab3 causes a preferential boost of synaptic activity encoded by intermediate spike frequencies.

Rab3 deletion lowers the $\mathrm{Ca}^{2+}$ sensitivity of release

To determine whether the decrease in synaptic responses in Rab3-deficient neurons is associated with a relative change in the apparent $\mathrm{Ca}^{2+}$ affinity of release, we titrated synaptic responses with increasing concentrations of extracellular $\mathrm{Cd}^{2+}(1-100$ $\mu \mathrm{M})$. If the lack of Rab3 increases the amount of $\mathrm{Ca}^{2+}$ required for release (i.e., decreases the apparent $\mathrm{Ca}^{2+}$ affinity of release), the apparent $\mathrm{Cd}^{2+}$ concentrations needed to inhibit release should be lower. Conversely, if a mutation decreases the amount of $\mathrm{Ca}^{2+}$ required for release (i.e., increases the apparent $\mathrm{Ca}^{2+}$ affinity of release), higher $\mathrm{Cd}^{2+}$ concentrations will be needed to
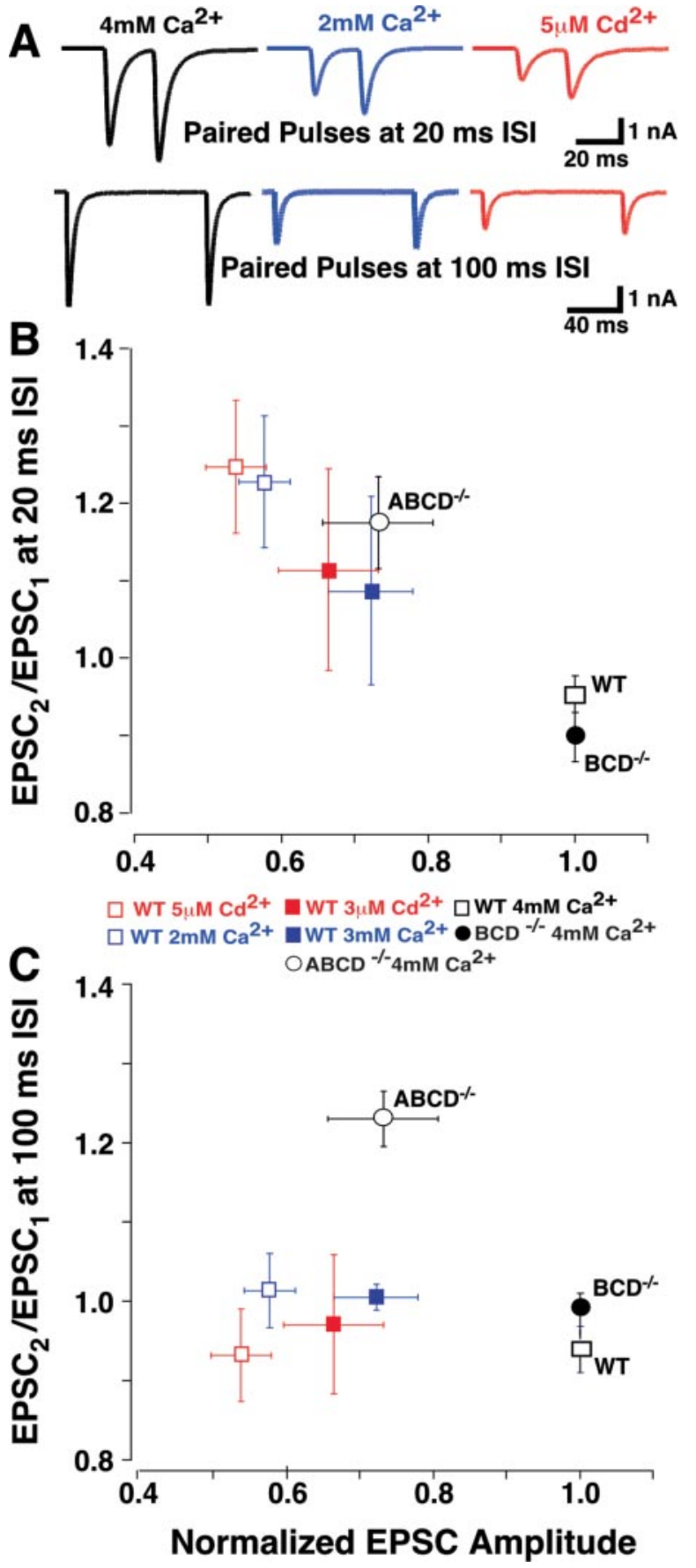

Figure 4. Rab3-dependent uncoupling of release probability and paired pulse behavior at intermediate interpulse intervals. $\boldsymbol{A}$, Exemplary traces of a wild-type neuron in response to paired pulses given at the interstimulus intervals (ISI) of $20 \mathrm{~ms}$ (top) and $100 \mathrm{~ms}$ (bottom) under conditions of varying $\mathrm{Ca}^{2+}$ influx: $4 \mathrm{~mm} \mathrm{Ca}{ }^{2+}$ (left, black traces), $3 \mathrm{~mm} \mathrm{Ca}^{2+}$ (middle, blue traces), and $4 \mathrm{~mm} \mathrm{Ca}^{2+} / 5 \mu \mathrm{m} \mathrm{Cd}{ }^{2+}$ (right, red traces). $\mathrm{Mg}^{2+}$ was $4 \mathrm{~mm}$ in all cases. Correlation of paired-pulse behavior at $20 \mathrm{~ms}(\boldsymbol{B})$ and $100 \mathrm{~ms}(\boldsymbol{C})$ interstimulus intervals and release probability in WT and Rab3-deficient mice. Release probabilities, as estimated from relative changes of EPSC amplitudes compared with control conditions $\left(4 \mathrm{~mm} \mathrm{Ca}^{2+}, 4 \mathrm{~mm} \mathrm{Mg}^{2+}\right.$; black open squares), were reduced by changing external $\mathrm{Ca}^{2+}$ concentrations ( $2-3 \mathrm{~mm}$; blue symbols) or by addition of $\mathrm{Cd}^{2+}$ ( 3 or $5 \mu \mathrm{m}$; red symbols) to the external solution. For comparison, the paired-pulse behavior of the Rab3-deficient (72\% of Rab3BCD ${ }^{-1-}$ synaptic amplitude; black filled circle) and Rab3a-containing (black open circle) synapses at control conditions are overlaid. Although at the $20 \mathrm{~ms}$ interval synaptic responses from wild-type neurons and in the Rab3 groups showed generally an increase in paired-pulse ratio with decreased release probability, little change of paired-pulse ratio was observed at $100 \mathrm{~ms}$ interstimulus intervals over a wide range of release probabilities in wild-type, whereas the Rab3-deficient synapses showed unusually strong facilitation at a $30 \%$ reduced release probability. 
A
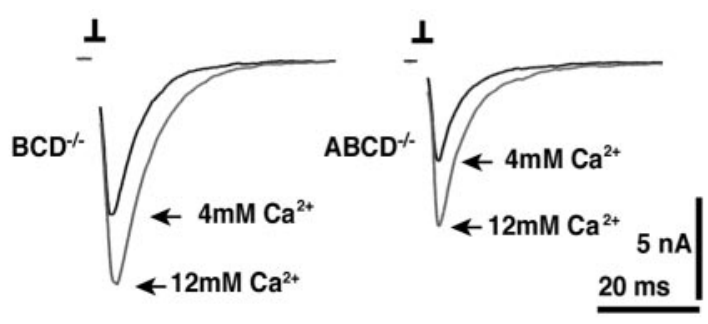
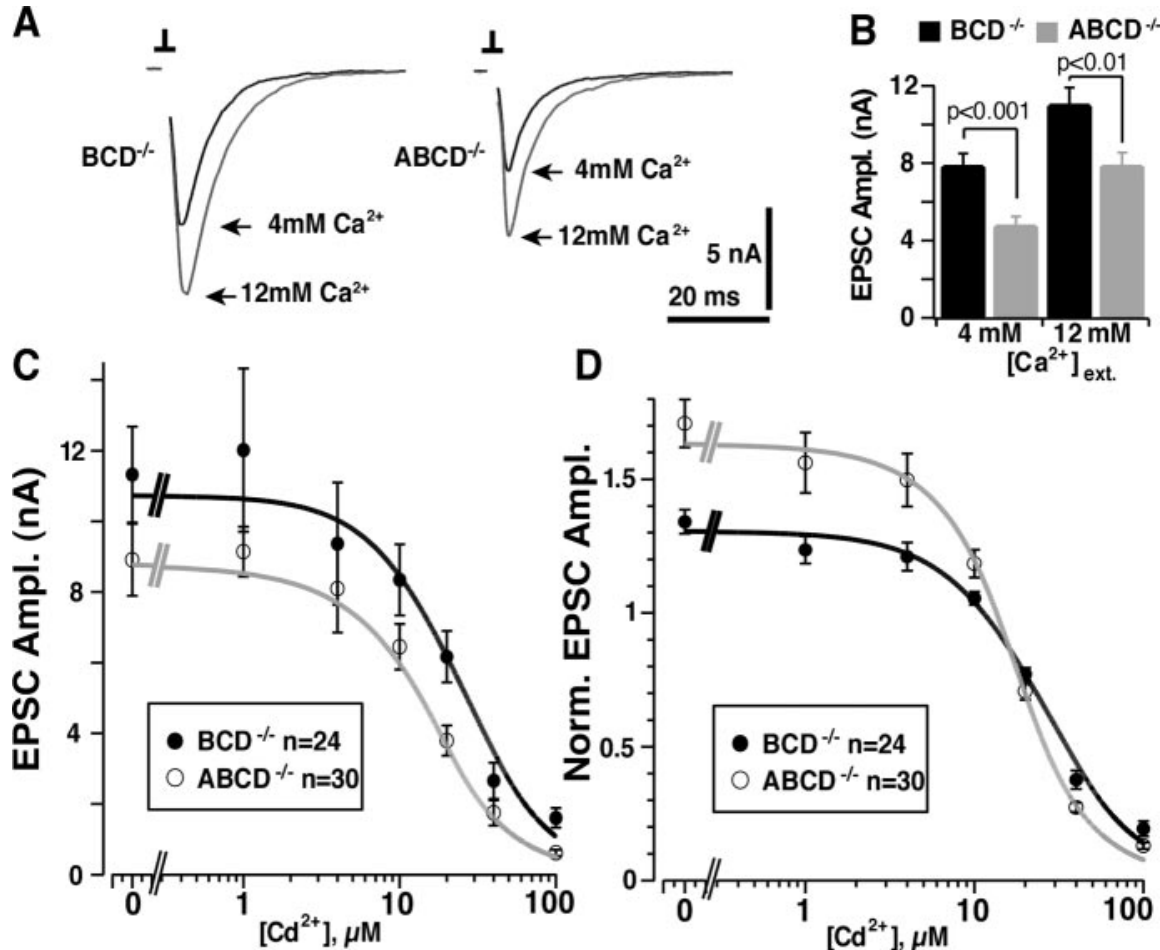

D

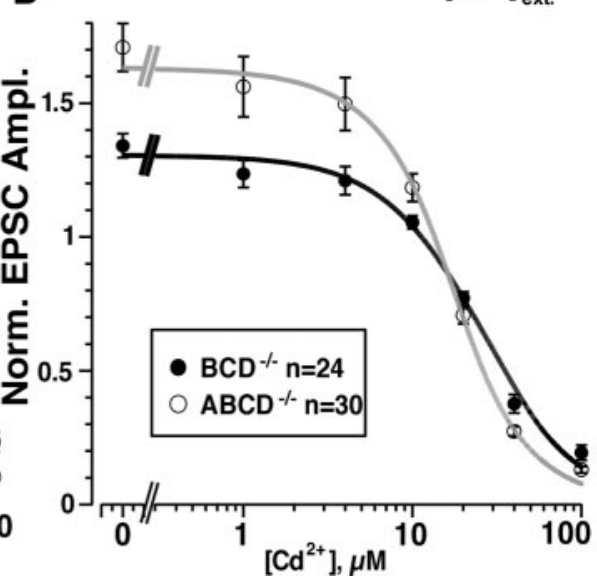

Figure 5. Decreased $\mathrm{Ca}^{2+}$ sensitivity in Rab3 K0 neurons. A, Sample traces of EPSCs from Rab3a-containing (top) and Rab3deficient (below) neurons evoked at $4 \mathrm{~mm}$ (black trace) and $12 \mathrm{~mm}$ (gray trace) $\mathrm{Ca}^{2+}$ concentrations. $\boldsymbol{B}$, Bar graph of mean absolute EPSC amplitudes (Ampl.) of Rab3a containing (Rab3BCD ${ }^{-1-}$; black bars) and Rab3-deficient (Rab3ABCD ${ }^{-1-}$; gray bars) at $4 \mathrm{~mm}$ $\mathrm{Ca}^{2+} / 4 \mathrm{~mm} \mathrm{Mg}^{2+}$ and at $12 \mathrm{~mm} \mathrm{Ca}{ }^{2+} / 1 \mathrm{~mm} \mathrm{Mg}^{2+}$. C, Titration of synaptic responses with increasing concentrations of extracellular $\mathrm{Cd}^{2+}(0-100 \mu \mathrm{m})$ to progressively block $\mathrm{Ca}^{2+}$ influx. Data shown are absolute EPSC sizes recorded in $12 \mathrm{~mm} \mathrm{Ca}^{2+}$ and $1 \mathrm{~mm} \mathrm{Mg}^{2+}$. D, Same data as in $\boldsymbol{C}$ but normalized to alternating control responses $\left(4 \mathrm{~mm} \mathrm{Ca}^{2+}, 4 \mathrm{~mm} \mathrm{Mg}{ }^{2+}\right)$. The $\mathrm{Ca}^{2+}$ cooperativity in the two groups was not significantly different $\left(\operatorname{Rab3ABCD}^{-1-}, 2.03 \pm 0.20 ; \operatorname{Rab}^{\circ} \mathrm{BCD}^{-1-}, 1.84 \pm 0.19\right.$ ).

inhibit release. To cover a wide range of release probabilities, the background $\mathrm{Ca}^{2+}$ concentration was elevated to near saturating levels ( $12 \mathrm{~mm}$ ). Generally, at all $\mathrm{Cd}^{2+}$ concentrations tested, we observed smaller absolute EPSC amplitudes in the Rab3-deficient neurons than in the Rab3A-containing neurons (Fig. $5 B, C$ ); this shows that elevation of $\mathrm{Ca}^{2+}$ does not rescue the lack of Rab3. However, when we fitted the data points with a Hill function to estimate the $\mathrm{Cd}^{2+}$ concentration required for half-maximal inhibition of release, significantly lower $\mathrm{Cd}^{2+}$ concentrations were required for inhibition of the Rab3-deficient neuronal release compared with Rab3a-containing neurons $\left(\mathrm{ABCD}^{-1-}, 17.3 \pm\right.$ $1.0 \mu \mathrm{M} \mathrm{Cd}^{2+}, n=30 ; \mathrm{BCD}^{-1-}, 29.1 \pm 2.8 \mu \mathrm{M} \mathrm{Cd}^{2+}, n=24 ; p<$ 0.001) (Fig. 5D).

This suggests that deletion of Rab3 decreases the overall apparent $\mathrm{Ca}^{2+}$ affinity of release. To confirm this, we measured the enhancement of synaptic responses induced by increased extracellular $\mathrm{Ca}^{2+}$ concentration $\left(12 \mathrm{mM} \mathrm{Ca}{ }^{2+} / 1 \mathrm{mM} \mathrm{Mg}^{2+}\right.$ vs $4 \mathrm{mM}$ $\left.\mathrm{Ca}^{2+} / 4 \mathrm{mM} \mathrm{Mg}^{2+}\right)$. We found that the relative potentiation of release in high $\mathrm{Ca}^{2+}$ was significantly larger in the Rab3-deficient than in Rab3a-containing neurons $\left(\mathrm{BCD}^{-1-}, 1.3 \pm 0.05\right.$-fold, $n=24 ; \mathrm{ABCD}^{-l-}, 1.7 \pm 0.09$-fold, $n=30 ; p<0.001$ ), consistent with the notion that deletion of all Rab3 isoforms decreases the apparent $\mathrm{Ca}^{2+}$ affinity of release. The increased $\mathrm{Ca}^{2+}$ sensitivity of release in the presence of Rab3 would move the efficiency of release to a more shallow region of the $\mathrm{Ca}^{2+}$-EPSC amplitude function and, in turn, lead to a smaller potentiation of the evoked response when $\mathrm{Ca}^{2+}$ is elevated.
Rab3 does not contribute to the release kinetics during evoked release

The data so far show that deletion of Rab3 effectively dampens the release probability of a subset of vesicles in the readily releasable pool, resulting in an unexpected alteration in short-term synaptic plasticity for a particular frequency domain. How is this Rab3dependent vesicle subpool defined? Vesicles may be characterized by displaying subpoolspecific kinetics of release. At room temperature, synaptic vesicles at hippocampal synapses are released with two time courses: a fast synchronous component, accounting for $\sim 80 \%$ of the charge of an EPSC, and a slow asynchronous component (Goda and Stevens, 1994). During repetitive stimulation the ratio is shifted progressively toward asynchronous release (Hagler and Goda, 2001; Pyott and Rosenmund, 2002). We tested whether Rab3 has any influence on the time course of release during repetitive stimulation by analyzing the relative size of synchronous and asynchronous release (Fig. 6). We detected no difference between Rab3-deficient and Rab3a-containing neurons, indicating that complete loss of Rab3 did not selectively affect one or the other component of release. This indicates that the proposed Rab3-induced heterogeneity within the RRP cannot be correlated with heterogeneity of vesicular release kinetics. Therefore, Rab3 action may not be associated with processes regulating the kinetics of the vesicular release.

\section{Vesicle priming in Rab3-deficient synapses}

We then focused on the well documented function of Rab proteins in the docking and tethering of vesicles to target membranes (for review, see Jahn et al., 2003). In principle, the parameter that would most likely be affected by lack of the tethering function is a change in vesicle-priming rates. Indeed, heterogeneity within the RRP has been demonstrated through dual time course of response recovery after activity-induced depletion of synaptic responses at the calyx of Held and at the cerebellar climbing fiber synapse (Silver et al., 1998; Sakaba and Neher, 2001a). We analyzed both $\mathrm{Ca}^{2+}$-dependent and $\mathrm{Ca}^{2+}$-independent pool refilling after depletion. Two consecutive pulses of hypertonic sucrose, separated by a variable interval, were used to measure $\mathrm{Ca}^{2+}$-independent refilling. The first pulse depletes the pool, and the second pulse monitors its reformation. We then plotted the amplitude of the second response as a percentage of the first response. Rab3-deficient neurons and Rab3a-containing neurons were indistinguishable in this assay, with both exhibiting a recovery time constant of $\sim 6 \mathrm{~s}$, similar to wild-type synapses (Rosenmund and Stevens, 1996) (Fig. 7A). This observation shows that vesicle priming at rest is not impaired after loss of Rab3.

In a second set of experiments, we investigated priming rates under conditions of elevated $\mathrm{Ca}^{2+}$, for example, after highfrequency stimulation. When the readily releasable pool of vesicles is depleted by an action potential train, the rate of pool refilling is faster than at resting $\mathrm{Ca}^{2+}$ concentrations (Stevens and Sullivan, 1998; Rhee et al., 2002). We depleted the primed vesicle 


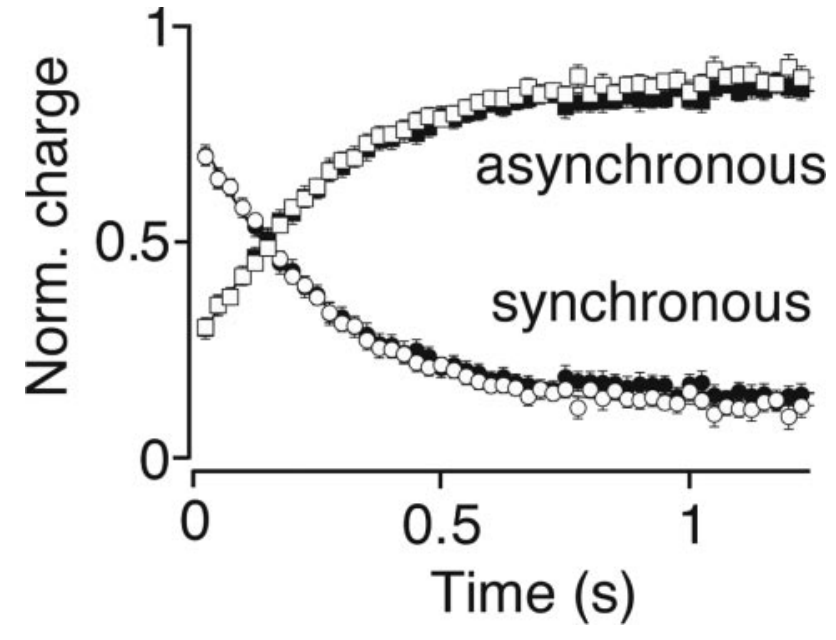

Figure 6. Loss of Rab3s does not affect timing of release. The plot shows the mean fraction of synchronous (circle symbols) and asynchronous (square symbols) release during an action potential train $(40 \mathrm{~Hz})$ in the Rab3a-containing (closed symbols) and Rab3-deficient (open symbols) neurons as a function of the stimulus number. The asynchronous component of the EPSC was extrapolated from the initial slope of the following integrated EPSC time course. The synchronous component was calculated by subtraction of the asynchronous component from the total charge transfer of an EPSC [for details, see Pyott and Rosenmund (2002)]. Norm., Normalized to sum of synchronous and asynchronous component.

pool with a $40 \mathrm{~Hz}$ train of action potentials in Rab3-deficient neurons and measured the recovery rate of synaptic responses by recording EPSCs induced by isolated action potentials (Fig. 7B). As expected, we found that synaptic responses of both Rab3acontaining and Rab3-deficient neurons recovered faster after action potential-driven depletion of the readily releasable pool (time constant, $\sim 1 \mathrm{~s}$ ) than after sucrose-induced depletion of this pool (time constant, $\sim 6 \mathrm{~s}$ ).

We then compared the recovery rate of the absolute amplitudes of each individual Rab3-deficient neuron (tau, $0.75 \pm 0.1 \mathrm{~s}$ ) and in Rab3a-containing neurons ( $\mathrm{tau}, 1.4 \pm 0.3 \mathrm{~s} ; p<0.05$ ) using initially a single exponential fit. We also calculated the Rab3-dependent component by subtraction of the Rab3deficient response from the control response (Fig. $7 B$ ). The time constant of the Rab3-dependent component was significantly larger (tau, $1.5 \mathrm{~s}$ ) than the time constant of refilling measured in the absence of Rab3. These data suggest that the Rab3-dependent component of release recovers at least twofold slower after depletion than the Rab3-independent component. Using previously published data from other synapses as a yard stick (Silver et al., 1998; Sakaba and Neher, 2001a), we probed whether this shift is based on a modification of one of the two kinetic components of pool recovery by replotting the normalized recovery phase of the EPSC (Fig. 7C). The recovery rate of the Rab3-deficient synapses was well fitted with a single exponential, resulting in a time constant of $0.48 \mathrm{~s}$, comparable with the recovery rate of the reluctant vesicles in the calyx and the fast recovery in the climbing fiber terminals. The recovery rate of the control neurons was then fitted with a double exponential function, setting one time constant fixed to $0.48 \mathrm{~s}$. This resulted in a second time constant of $2.2 \mathrm{~s}$. The weight of the fast recovery was $\sim 40 \%$, whereas the slow recovery contributed $\sim 60 \%$. Thus, in the absence of Rab3, EPSCs recover fast, whereas Rab3 is required for the presence of an additional slowly recovering (tau, $\sim 2 \mathrm{~s}$ ) response component. These data are consistent with the idea of an overall functional heterogeneity of primed vesicles and implicates the essential role of Rab3 in this heterogeneity.

\section{Discussion}

Rab3 proteins are abundant GTP-binding proteins of synaptic vesicles (Sudhof, 2004). In a recent study, we showed that deletion of all four Rab3 isoforms, but not of any combination of three Rab3 isoforms, causes lethality in mice, results in a $\sim 30 \%$ decrease in $\mathrm{Ca}^{2+}$-evoked neurotransmitter release, and causes an equivalent decline in the synaptic and vesicular release probabilities $P_{\mathrm{r}}$ and $P_{\mathrm{vr}}$ (Schluter et al., 2004). This simultaneous lethality of the quadruple Rab3 KO with a surprisingly discrete impairment in presynaptic function raised two questions: which step in the synaptic vesicle cycle does Rab3 deletion affect so as to account for the discrete modification in neurotransmitter release, and why is such a relatively small decrease in release lethal in consequence?

Although we can only speculate about the origin of the lethal consequences (see below), the present study allows for quite a precise determination of the role of Rab3 on the dynamics of vesicle release. Our data suggest that Rab3 acts on a subset of vesicles in the RRP to increase their release probability, a function that we call "superpriming." The evidence of heterogeneity of synaptic vesicle function is based on the following arguments. We see an initial reduction in the EPSC amplitude but no change in vesicle pool size in Rab3-deficient neurons (Schluter et al., 2004). Therefore, the average release probability is reduced, and Rab3 targets its effect within the RRP. Second, we measured a higher paired-pulse ratio at $\mathrm{AP}$ frequencies of $10-50 \mathrm{~Hz}$ than predicted from a mean reduction of release probability, suggesting that lack of Rab3 reduces release probability unequally within the RRP (Figs. 1, 3, 4). Third, we see different repriming rates for the Rab3-dependent and -independent components, suggesting that Rab3 modulation introduces a rate-limiting step in priming (Fig. 7). Fourth, we noticed a significant shift in the apparent $\mathrm{Ca}^{2+}$ sensitivity of the Rab3-dependent versus -independent components (Fig. 5). This suggests that Rab3 helps to increase the efficiency of release. All of these features imply that the vesicles "superprimed" by Rab3 are the first to undergo exocytosis when a nerve terminal is invaded by trains of action potentials. However, because they are reprimed relatively slowly after exocytosis, their effect on the response size ceases rather quickly during ongoing synaptic use.

What are the characteristics of the Rab3-dependent and -independent pools of vesicles? The time course of release is similar in both vesicle populations, because neither the time course of individual EPSCs nor the shift from more synchronous to asynchronous release during repetitive stimulation is changed after removal of Rab3 (Schluter et al., 2004). This finding is consistent with a previous study showing that release probabilities of the asynchronous and synchronous components are similar (Otsu et al., 2004). However, the superprimed pool shares important similarities with the fast-depleting vesicles of the calyx of Held. Both the superprimed pool and the fast-depleting vesicles constitute $40-50 \%$ of the RRP, they have a relatively high release probability, and are replenished with a time constant of 2-5 s (Sakaba and Neher, 2001a,b). In contrast, the Rab3-independent vesicles and the slowly depleted vesicles of the calyx of Held have a reduced release probability but are replenished with a time constant of 200-500 ms. Significantly, the time course of an EPSC does not change regardless of whether the synapse is stimulated in a naive state or after partial replenishment of the reluctant pool [referred to as slowly depleting pool in the study by Sakaba and Neher (2001a,b)]. This states that pool heterogeneity based on differences in release probability are not correlated with 

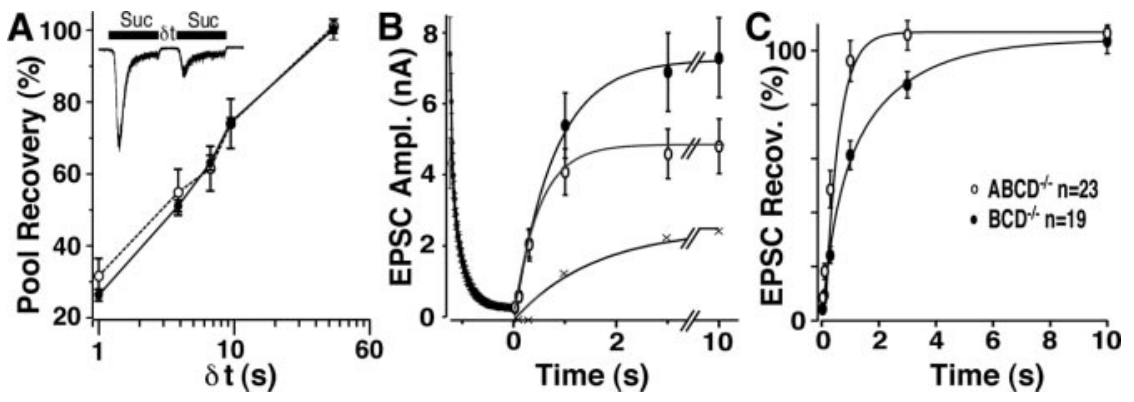

Figure 7. Slow recovery of the Rab3-dependent response component from action potential train-induced pool depletion. $A$, Recovery of the readily releasable pool measured as the release triggered by hypertonic sucrose (Suc). Two successive sucrose pulses ( $4 \mathrm{~s}$ ) were applied separated by an interval of 1-100 s, and the size of the second response is measured as a percentage of the first (note the semilogarithmic representation; $n=10-13$ for each time point and group). $\boldsymbol{B}$, Recovery of the readily releasable pool measured as the release triggered by action potentials. The readily releasable pool was first depleted by a $1.25 \mathrm{~s}$ stimulation at $40 \mathrm{~Hz}$, and then the recovery was followed by measuring synaptic responses after $0.1,0.3$, 1,3 , and $10 \mathrm{~s}$. Responses are displayed as absolute amplitudes (Ampl.). Data shown are means \pm SEMs. C, Normalized (to initial EPSC) pool recovery of $A B C D-/-$ (open symbols) and $B C D-/-$ (closed symbols) neurons. Exponential fitting of recovery were performed as described in Results.

heterogeneity in the time course of triggered release ( $\mathrm{Wu}$ and Borst, 1999).

This study introduces a new function for Rab proteins, namely the boosting of the efficiency of the $\mathrm{Ca}^{2+}$-triggered release by modifying already primed vesicles, thereby introducing heterogeneity in the primed vesicle pool. We can think of two alternatives but not exclusive mechanisms for this Rab3-induced superpriming effect. First, Rab3 may serve to direct vesicles to release sites with intrinsically high release probability, for example next to $\mathrm{Ca}^{2+}$ channels, because close proximity of vesicles to $\mathrm{Ca}^{2+}$ channels increases their release probability (Meinrenken et al., 2002). Second,, Rab3 may function to recruit or enhance binding of additional proteins to the release machinery that make $\mathrm{Ca}^{2+}$-triggered release more efficient. Based on the observed phenotype, we think that the two best described Rab3 effector molecules rabphilin and Rim1 (Shirataki et al., 1993; Wang et al., 1997) are unlikely to be involved in the Rab3 function described above. Removal of rabphilin in mice has no detectable influence on synaptic transmission (Schluter et al., 1999), and deletion of Rim 1 in mice causes a change of other parameters compared with Rab3-deficient mice (Schoch et al., 2002; Calakos et al., 2004). Comparable with the Rab3 quadruple KOs, the Rim 1 KO neurons display a 50\% decrease in EPSC size (Calakos et al., 2004), but this is accompanied by a parallel reduction in RRP size and protein levels of the priming factor Munc13-1, which are not observed in the Rab3-deficient mice. Furthermore, a shift in the release probability and apparent $\mathrm{Ca}^{2+}$ affinity was only seen in Rab3 quadruple KOs, whereas only the RIM1 KOs showed a prominent decrease in the asynchronous component of release. Although both Rim1 KO and Rab3 quadruple KO neurons display increased paired-pulse facilitation, close inspection reveals that the effect is evident at different frequencies in the two KO mouse models (Rim1 short interpulse intervals, Rab3 intermediate interpulse intervals). These phenotypical differences between Rim1 KO, rabphilin KO, and Rab3 quadruple KO suggests that Rab3 acts on yet undefined target(s). Furthermore, a comparison of the Rab3 and Rim1 KO models suggests that Rim1 acts during initial priming to globally increase synaptic release probability, whereas Rab3 enters in a secondary priming step to cause an increased efficiency of triggered release by targeting a sub- set of primed vesicles. A possible additional effector of Rab3 could be Synapsin I, which was shown to bind to Rab3a and modulate its function in vitro (Giovedi et al., 2004). Indeed, the paired-pulse ratio at a CA1 pyramidal cell synapse is increased in the synapsin I KO mice (Rosahl et al., 1993). Despite the lack of change of the number of docked vesicles, the absence of synapsin I causes a reduction of the immediately releasable pool ( $\mathrm{Li}$ et al., 1995; Ryan et al., 1996). In addition to its function in regulating the reserve pool of vesicles, these results suggest a potential function of synapsin I in regulating release probability, potentially via Rab3.

How does the function of Rab3 in boosting of a subset of vesicles translate in synaptic encoding pattern? The $30 \%$ decrease in initial synaptic responses in the Rab3-deficient neurons can probably be compensated by a vertebrate nervous system. It seems feasible that moderate and uniform change in synaptic strength can readily be adjusted by homeostatic mechanisms (Panzeri et al., 2001; Turrigiano and Nelson, 2004).

What is likely to be of impact in synaptic coding is not the $30 \%$ decrease as such but the fact that the relative weakness of Rab3-deficient synapses is dependent on the frequency and the history of synaptic activity. Removal of Rab3 mainly affects the first few spikes of a train of action potentials, and this cannot simply be compensated by adding more synapses or adjustment of synaptic strength of individual synapses. A change in synaptic strength during spike trains may have strong impact on many aspects of synaptic encoding, one of such examples may be the precision of timing of neuronal excitation (Silberberg et al., 2004) or the cortical processing of sensory information. Studies correlating sensory inputs with firing patterns in the processing cortical neuron show that the delay between a sensory stimulus (hence synaptic input) and the first postsynaptic spike carried $80-90 \%$ of the total sensory information (Panzeri et al., 2001). Based on this, the action of Rab3 would make first spike delay much more precise. A third example is the role in auditory processing: precise regulation of short-tem plasticity features participates in distinguishing static from dynamic qualities of the auditory signal process in the primary auditory cortex (Atzori et al., 2001).

How can these changes in short-tem plasticity be of importance for the survival of mice? One obvious speculation is that the short-term plasticity profoundly disturb the neuronal network responsible for respiratory rhythmogenesis (Schluter et al., 2004) or, alternatively, the lack of Rab3 function in peripheral nervous system, directly at the neuromuscular junction of the diaphragm might cause perinatal lethality in the Rab3-deficient mice. Importantly, Rab3a is abundant at the neuromuscular junction (Mizoguchi et al., 1992).

Although our analysis revealed that Rab3 functions in a much more discreet manner than is expected from its general role in vesicle trafficking, the deleterious impact of loss of Rab3 function, however, is powerful evidence for the general importance of this function in synaptic encoding and for precise short-term plasticity pattern in shaping temporal properties in neuronal networks in general. 


\section{References}

Atzori M, Lei S, Evans DI, Kanold PO, Phillips-Tansey E, McIntyre O, McBain CJ (2001) Differential synaptic processing separates stationary from transient inputs to the auditory cortex. Nat Neurosci 4:1230-1237.

Baldini G, Scherer PE, Lodish HF (1995) Nonneuronal expression of Rab3A: induction during adipogenesis and association with different intracellular membranes than Rab3D. Proc Natl Acad Sci USA 92:4284-4288.

Bekkers JM, Stevens CF (1991) Excitatory and inhibitory autaptic currents in isolated hippocampal neurons maintained in cell culture. Proc Natl Acad Sci USA 88:7834-7838.

Blatow M, Caputi A, Burnashev N, Monyer H, Rozov A (2003) $\mathrm{Ca}^{2+}$ buffer saturation underlies paired pulse facilitation in calbindin-D28kcontaining terminals. Neuron 38:79-88.

Calakos N, Schoch S, Sudhof TC, Malenka RC (2004) Multiple roles for the active zone protein RIMlalpha in late stages of neurotransmitter release. Neuron 42:889-896.

Edmonds B, Reyes R, Schwaller B, Roberts WM (2000) Calretinin modifies presynaptic calcium signaling in frog saccular hair cells. Nat Neurosci 3:786-790.

Fernandez-Chacon R, Konigstorfer A, Gerber SH, Garcia J, Matos MF, Stevens CF, Brose N, Rizo J, Rosenmund C, Sudhof TC (2001) Synaptotagmin I functions as a calcium regulator of release probability. Nature 410:41-49.

Giovedi S, Darchen F, Valtorta F, Greengard P, Benfenati F (2004) Synapsin is a novel Rab3 effector protein on small synaptic vesicles. II. Functional effects of the Rab3A-synapsin I interaction. J Biol Chem 279:43769-43779.

Goda Y, Stevens CF (1994) Two components of transmitter release at a central synapse. Proc Natl Acad Sci USA 91:12942-12946.

Hagler Jr DJ, Goda Y (2001) Properties of synchronous and asynchronous release during pulse train depression in cultured hippocampal neurons. J Neurophysiol 85:2324-2334.

Jahn R, Lang T, Sudhof TC (2003) Membrane fusion. Cell 112:519-533.

Li L, Chin LS, Shupliakov O, Brodin L, Sihra TS, Hvalby Ø, Jensen V, Zheng D, McNamara JO, Greengard P, Andersen P (1995) Impairment of synaptic vesicle clustering and of synaptic transmission, and increased seizure propensity, in synapsin I-deficient mice. Proc Natl Acad Sci USA 92:9235-9239.

Meinrenken CJ, Borst JG, Sakmann B (2002) Calcium secretion coupling at calyx of held governed by nonuniform channel-vesicle topography. J Neurosci 22:1648-1667.

Mintz IM, Sabatini BL, Regehr WG (1995) Calcium control of transmitter release at a cerebellar synapse. Neuron 15:675-688.

Mizoguchi A, Arakawa M, Masutani M, Tamekane A, Yamaguchi H, Minami N, Takai Y, Ide C (1992) Localization of smg p25A/rab3A p25, a small GTP-binding protein, at the active zone of the rat neuromuscular junction. Biochem Biophys Res Commun 186:1345-1352.

Otis T, Zhang S, Trussell LO (1996) Direct measurement of AMPA receptor desensitization induced by glutamatergic synaptic transmission. J Neurosci 16:7496-7504.

Otsu Y, Shahrezaei V, Li B, Raymond LA, Delaney KR, Murphy TH (2004) Competition between phasic and asynchronous release for recovered synaptic vesicles at developing hippocampal autaptic synapses. J Neurosci 24:420-433.

Panzeri S, Petersen RS, Schultz SR, Lebedev M, Diamond ME (2001) The role of spike timing in the coding of stimulus location in rat somatosensory cortex. Neuron 29:769-777.

Pereira-Leal JB, Hume AN, Seabra MC (2001) Prenylation of Rab GTPases: molecular mechanisms and involvement in genetic disease. FEBS Lett 498:197-200

Pyott SJ, Rosenmund C (2002) The effects of temperature on vesicular supply and release in autaptic cultures of rat and mouse hippocampal neurons. J Physiol (Lond) 539:523-535.

Rhee J-S, Betz A, Pyott S, Reim K, Varoqueaux F, Augustin I, Hesse D, Südhof T, Takahashi M, Rosenmund C, Brose N (2002) b-Phorbol ester and diacylglycerol-induced augmentation of transmitter release is mediated by Munc13s and not by PKC. Cell 108:121-133.

Rosahl TW, Geppert M, Spillane D, Herz J, Hammer RE, Malenka RC, Südhof TC (1993) Short-term synaptic plasticity is altered in mice lacking synapsin I. Cell 75:661-670.

Rosenmund C, Stevens CF (1996) Definition of the readily releasable pool of vesicles at hippocampal synapses. Neuron 16:1197-1207.
Rozov A, Burnashev N, Sakmann B, Neher E (2001) Transmitter release modulation by intracellular $\mathrm{Ca}^{2+}$ buffers in facilitating and depressing nerve terminals of pyramidal cells in layer $2 / 3$ of the rat neocortex indicates a target cell-specific difference in presynaptic calcium dynamics. J Physiol (Lond) 531:807-826.

Ryan TA, Li L, Chin LS, Greengard P, Smith SJ (1996) Synaptic vesicle recycling in synapsin I knock-out mice. J Cell Biol 134:1219-1227.

Sakaba T, Neher E (2001a) Quantitative relationship between transmitter release and calcium current at the calyx of held synapse. J Neurosci 21:462-476.

Sakaba T, Neher E (2001b) Calmodulin mediates rapid recruitment of fastreleasing synaptic vesicles at a calyx-type synapse. Neuron 32:1119-1131.

Schluter OM, Schnell E, Verhage M, Tzonopoulos T, Nicoll RA, Janz R, Malenka RC, Geppert M, Sudhof TC (1999) Rabphilin knock-out mice reveal that rabphilin is not required for rab3 function in regulating neurotransmitter release. J Neurosci 19:5834-5846.

Schluter OM, Khvotchev M, Jahn R, Sudhof TC (2002) Localization versus function of Rab3 proteins. Evidence for a common regulatory role in controlling fusion. J Biol Chem 277:40919-40929.

Schluter OM, Schmitz F, Jahn R, Rosenmund C, Sudhof TC (2004) A complete genetic analysis of neuronal Rab3 function. J Neurosci 24:6629-6637.

Schoch S, Castillo PE, Jo T, Mukherjee K, Geppert M, Wang Y, Schmitz F, Malenka RC, Sudhof TC (2002) RIMlalpha forms a protein scaffold for regulating neurotransmitter release at the active zone. Nature 415:321-326.

Shirataki H, Kaibuchi K, Sakoda T, Kishida S, Yamaguchi T, Wada K, Miyazaki M, Takai Y (1993) Rabphilin-3A, a putative target protein for smg p25A/rab3A p25 small GTP-binding protein related to synaptotagmin. Mol Cell Biol 13:2061-2068.

Silberberg G, Wu C, Markram H (2004) Synaptic dynamics control the timing of neuronal excitation in the activated neocortical microcircuit J Physiol (Lond) 556:19-27.

Silver RA, Momiyama A, Cull-Candy SG (1998) Locus of frequencydependent depression identified with multiple-probability fluctuation analysis at rat climbing fibre-Purkinje cell synapses. J Physiol (Lond) 510:881-902.

Sippy T, Cruz-Martin A, Jeromin A, Schweizer FE (2003) Acute changes in short-term plasticity at synapses with elevated levels of neuronal calcium sensor-1. Nat Neurosci 6:1031-1038.

Stevens CF, Sullivan JM (1998) Regulation of the readily releasable vesicle pool by protein kinase C. Neuron 21:885-893.

Stevens CF, Wesseling JF (1998) Activity-dependent modulation of the rate at which synaptic vesicles become available to undergo exocytosis. Neuron 21:415-424.

Sudhof TC (2004) The synaptic vesicle cycle. Annu Rev Neurosci 27:509-547.

Touchot N, Chardin P, Tavitian A (1987) Four additional members of the ras gene superfamily isolated by an oligonucleotide strategy: molecular cloning of YPT-related cDNAs from a rat brain library. Proc Natl Acad Sci USA 84:8210-8214.

Trommershauser J, Schneggenburger R, Zippelius A, Neher E (2003) Heterogeneous presynaptic release probabilities: functional relevance for short-term plasticity. Biophys J 84:1563-1579.

Turrigiano GG, Nelson SB (2004) Homeostatic plasticity in the developing nervous system. Nat Rev Neurosci 5:97-107.

von Gersdorff H, Schneggenburger R, Weis S, Neher E (1997) Presynaptic depression at a calyx synapse: the small contribution of metabotropic glutamate receptors. J Neurosci 17:8137-8146.

Wang Y, Okamoto M, Schmitz F, Hofmann K, Sudhof TC (1997) Rim is a putative Rab3 effector in regulating synaptic-vesicle fusion. Nature 388:593-598.

Wu LG, Borst JG (1999) The reduced release probability of releasable vesicles during recovery from short-term synaptic depression. Neuron 23:821-832.

$\mathrm{Xu}$ J, Wu LG (2005) The decrease in the presynaptic calcium current is a major cause of short-term depression at a calyx-type synapse. Neuron 46:633-645.

Zahraoui A, Touchot N, Chardin P, Tavitian A (1989) The human Rab genes encode a family of GTP-binding proteins related to yeast YPT1 and SEC4 products involved in secretion. J Biol Chem 264:12394-12401.

Zucker RS, Regehr WG (2002) Short-term synaptic plasticity. Annu Rev Physiol 64:355-405. 